\title{
A FUNCTIONAL EQUATION PROPOSED BY R. BELLMAN
}

\author{
E. G. STRAUS
}

R. Bellman (Bull. Amer. Math. Soc. vol. 64 (1958) p. 178, problem 14) has posed (essentially) the following problem:

Let $f\left(u_{0}, u_{1}, \cdots, u_{n}\right)$ be analytic for $u_{0} \neq 0$ and satisfy the functional equation

(1) $f\left(u v,(u v)^{\prime}, \cdots,(u v)^{(n)}\right)=f\left(u, u^{\prime}, \cdots, u^{(n)}\right)+f\left(v, v, \cdots, v^{(n)}\right)$

for arbitrary $x$ and arbitrary nonzero $n$ times differentiable functions $u(x), v(x)$. What is the form of $f$ for general $n$ ?

At the suggestion of R. Bellman and of N. J. Fine (who has given another solution of this problem), ${ }^{1}$ we give here a solution which seems to stress the proper setting of the problem.

Set $u=e^{s}, v=e^{t}$; then we can write

$$
f\left(u, u^{\prime}, \cdots, u^{(n)}\right)=g\left(s, s^{\prime}, \cdots, s^{(n)}\right),
$$

and the functional equation (1) becomes

(2) $g\left(s+t, s^{\prime}+t^{\prime}, \cdots, s^{(n)}+t^{(n)}\right)=g\left(s, \cdots, s^{(n)}\right)+g\left(t, \cdots, t^{(n)}\right)$

for arbitrary $x$, and arbitrary $n$ times differentiable $s, t$.

Applying (2) to the functions

$$
s=\sum_{k=0}^{n} \frac{s_{k}\left(x-x_{0}\right)^{k}}{k !}
$$

we get for $G(s)=g\left(s, s^{\prime}, \cdots, s^{(n)}\right)$ that at $x=x_{0}$

$$
\begin{aligned}
G(s)= & G\left(\sum \frac{s_{k}\left(x-x_{0}\right)^{k}}{k !}\right)=\sum G\left(\frac{s_{k}\left(x-x_{0}\right)^{k}}{k !}\right) \\
= & g\left(s_{0}, 0, \cdots, 0\right)+g\left(0, s_{1}, 0, \cdots, 0\right)+\cdots \\
& +g\left(0,0, \cdots, 0, s_{n}\right) \\
= & \sum_{k=0}^{n} g_{k}\left(s_{k}\right) .
\end{aligned}
$$

As a result of (2) we see that each of the functions $g_{k}\left(s_{k}\right)$ is additive. Summing up we have the following 1959.

Received by the editors December 29, 1958 and, in revised form, February 2,

${ }^{1}$ Solutions have also been given by W. F. Trench and by J. Aczél and M. Hosszú. 
Theorem 1. Let $f\left(x, u_{0}, u_{1}, u_{2}, \cdots, u_{n}\right)$ be a function of $n+2$ variables so that

$$
\begin{aligned}
f\left(x, u v,(u v)^{\prime}, \cdots,\right. & \left.(u v)^{(n)}\right) \\
& =f\left(x, u, u^{\prime}, \cdots, u^{(n)}\right)+f\left(x, v, v^{\prime}, \cdots, v^{(n)}\right)
\end{aligned}
$$

for all $x$ and all nonzero $n$ times differentiable $u, v$. Then

$$
f\left(x, u, u^{\prime}, \cdots, u^{(n)}\right)=\sum_{k=0}^{n} f_{k}\left(x, d^{k} \log u / d x^{k}\right)
$$

where each $f_{k}(x, y)$ is additive in $y$. In particular, under any one of the usual conditions (e.g., continuity, measurability, boundedness) on $f_{k}(x, y)$ as a function of $y$ we obtain

$$
f\left(x, u^{\prime}, \cdots, u^{(n)}\right)=\sum_{k=0}^{n} a_{k}(x) \frac{d^{k} \log u}{d x^{k}} .
$$

In Bellman's problem $f$ did not directly depend on $x$ so that the $a_{k}$ in (5) would be constant.

We can now make several observations. The first (whose formulation is due to R. Steinberg) is that the function $u v$ in (1) and (4) could be replaced by $\psi(u, v)=\phi_{1}\left(\phi_{2}(u)+\phi_{2}(v)\right)$, where $\phi_{i}$ is an $n$ times differentiable function and $\phi_{2}^{\prime} \neq 0$, where the range of $\phi_{2}$ is the entire real line. So we have the following

Theorem 2. Let $f\left(x, u_{0}, u_{1}, \cdots, u_{n}\right)$ be a function of $n+2$ variables so that

$$
\begin{aligned}
f\left(x, \psi(u, v),(\psi(u, v))^{\prime}, \cdots,\right. & \left.(\psi(u, v))^{(n)}\right) \\
& =f\left(x, u, \cdots, u^{(n)}\right)+f\left(x, v, \cdots, v^{(n)}\right) .
\end{aligned}
$$

Then

$$
f\left(x, u, u^{\prime}, \cdots, u^{(n)}\right)=\sum_{k=0}^{n} f_{k}\left(x, \frac{d^{k} \phi_{2}(u)}{d x^{k}}\right)+a(x)
$$

where $f_{k}(x, y)$ is additive in $y$, and under suitable regularity conditions

$$
f\left(x, u, u^{\prime}, \cdots, u^{(n)}\right)=\sum_{k=0}^{n} a_{k}(x) \frac{d^{k} \phi_{2}(u)}{d x^{k}}+a(x) .
$$

Proof. We set $s=\phi_{2}(u), t=\phi_{2}(v)$. Then we can write

$$
f\left(x, u, u^{\prime}, \cdots, u^{(n)}\right)=g\left(x, s, \cdots, s^{(n)}\right)(=g(x, s) \text { for short })
$$




$$
f\left(x, \phi_{1}(s), \cdots, \phi_{1}(s)^{(n)}\right)=h\left(x, s, \cdots, s^{(n)}\right)(=h(x, s) \text { for short }) .
$$

Thus (6) becomes

(6) $h\left(x, s+t, \cdots, s^{(n)}+t^{(n)}\right)=g\left(x, s, \cdots, s^{(n)}\right)+g\left(x, t, \cdots, t^{(n)}\right)$

for all $n$ times differentiable functions $s, t$ at a point $x$.

For $t=0$ we obtain

$$
h(x, s)=g(x, s)+g(x, 0)=g(x, s)+a(x)
$$

and for

$$
k(x, s)=g(x, s)-a(x)=h(x, s)-2 a(x)
$$

equation $\left(6^{\prime}\right)$ becomes

$$
k(x, s+t)=k(x, s)+k(x, t) .
$$

This equation is analogous to (2) and leads to (7). We can now use (7) to analyze the functions $\phi_{1}$ and $a$ to obtain

$$
\phi_{1}(u)=\phi_{2}^{-1}(u+c) \text { and } a(x)=f_{0}(x, c)
$$

for some constant $c$.

Finally the fact that differentiation operators only were used was merely due to the fact they satisfy relations of the form

$$
D^{k} f(s)=g\left(s, D s, D^{2} s, \cdots, D^{k} s\right)
$$

for all $k$ times differentiable $s$ and arbitrarily given $k$ times differentiable $f$. We could replace these $D^{k}$ by arbitrary linear operators $T_{1}, \cdots, T_{n}$ which satisfy identities of the form

$$
\left(T_{i} \phi_{1}(s)\right)(x)=g_{i}\left(s, T_{1} s, \cdots, T_{n} s\right) \quad i=1, \cdots, n
$$

for all $x$, all $s$ in the prescribed function space and the function $\phi_{1}$ as in Theorem 2.

It is quite unnecessary to restrict the operator $f\left(u, u^{\prime}, \cdots, u^{(n)}\right)$ to dependence on a finite number of derivatives; the same result would hold if we had a differential operator of infinite order, or more generally, if we had operators $T_{i}$ satisfying (8) and so that for every $x$ there exist functions $s_{i}$ with $\left(T_{i} s_{j}\right)(x)=\delta_{i j}$.

We forego the statement of this theorem in all its gruesome generality.

University of California, Los Angeles 